\title{
Comparative Analysis of Pre-Germination and Post-Germination Inoculation Treatments of Zea mays L. to Mitigate Chromium Toxicity in Cr-Contaminated Soils
}

\author{
Sabiha Habib, Hadia-e-Fatima, Ambreen Ahmed* \\ Department of Botany, University of the Punjab, Quaid-e-Azam Campus, Lahore, Pakistan
}

Received: 17 August 2017

Accepted: 25 December 2017

\begin{abstract}
The release of heavy metals in the environment is a serious threat causing health hazards to living beings. Hence, it is essential to remediate chemical contamination for a safe environment. Bioremediation is considered one of the most cost-effective and sustainable agricultural techniques, in contrast with other conventional methods to reduce chromium toxicity in agricultural lands polluted with chromium, as it is a natural way to mitigate the toxic effects of hexavalent chromium with simultaneous amelioration in the growth of plants. In the current study, an attempt was made to reduce toxicity of chromium by using six plant growth-promoting chromium-resistant bacteria (Bacillus pumilus (ALa), Bacillus atrophaeus (BL2), Bacillus cereus (AR), Staphylococcus lentus (E3), T2aii and W6ii) for enhancing the growth of Zea mays L. in soil contaminated with chromium. In this regard, a pot experiment was conducted with pre-germination and post-germination inoculation treatments to Zea mays seeds in the presence of chromium stress, i.e., 200,400 , and $600 \mu \mathrm{g} / \mathrm{ml}$. Our results have shown that toxicity of chromium caused a reduction in photosynthetic pigments and protein content together with reduction in growth parameters of plants, while treatments with chromium-resistant PGPB significantly enhanced chromium tolerance in treated plants compared with non-inoculated treatments in the presence of chromium stress. The present investigation suggests that applying post-germination inoculation treatments is an effective technique for improved plant growth and heavy metal alleviation in metal-contaminated soil. Thus, our current work revealed an incentive approach toward the green revolution in the age of industrialization by exploring beneficial chromium-tolerant auxin-producing microbes.
\end{abstract}

Keywords: bioremediation, pre-germination, post-germination, Zea mays, PGPB

*e-mail: ambreenahmed1@hotmail.com 


\section{Introduction}

Soil is a collection of air, water, liquids, gases, metals, minerals, organic matter, and countless microorganisms that together maintain life on this planet by playing their role in the production of primary producers. But conversely, the presence of heavy metals due to the addition of various hazardous chemicals deposited in the soil by several anthropogenic activities exerts negative impact on the soil [1]. Therefore, toxicity of heavy metals in the soil is an important issue since soil is the medium for the growth of plants and these plants are the primary source of food for both humans and animals [2]. Chromium is regarded as the second most abundant metal in the earth's crust, having two oxidation states: $\mathrm{Cr}$ (III) and $\mathrm{Cr}$ (VI). Hexavalent chromium (Cr (VI)) is more toxic, highly permeable, and mobile, and can enter into food chains involving plants, animals, and humans [2]. Chromium toxicity causes inhibition of photosynthesis and disturbs the respiratory tract, sometimes leading to plant death [3]. The accumulation of chromium can also affect plant height, cause nutrient imbalance, inhibit microbial activities in soil, and is involved in the formation of some reactive oxygen species (ROS) resulting in oxidative stress in plants [4].

Many techniques have been introduced to prevail over the detrimental effects of heavy metal contamination in the physical, chemical, and biological environmental processes [5]. Among different remediation techniques, the biological approach is considered most important, including bioremediation, which is a useful and practical technique to clean up and minimize metal toxicity in soil and thus help in sustainable production of plants [6]. Over the last few decades, the mutualistic relationship between plants and bacteria has been studied by many scientists. Bacteria exert positive effects on the growth of plants. Similarly, plants choose an ideal microbiome to colonize their favorable bacterial population around plant roots [7]. Plant growth-stimulating bacteria have specific properties such as the production of phytohormones, secretion of secondary metabolites, mineral solubilization, and siderophore production that make it possible for them to promote productivity of plants. These bacteria also help in saving the plants from several environmental stresses along with heavy metal stress in soil, resulting in the retardation of plant growth [8]. The use of such beneficial bacteria is considered an environmentally benign and efficient method for crop improvement rather than the use of synthetic fertilizers in agricultural lands, which have many deleterious impacts on the environment [9]. Applying these chemical fertilizers to improve crop yield is costly, so the application of PGPRs having growth promotion potential as well as chromium tolerance is considered a promising technique for obtaining better yield [10]. In the proposed study, the plant chosen for experiment was Zea mays, which is the third most important cereal crop after wheat and rice in Pakistan and is used as a staple food for humans as well as animals worldwide [5]. The aim of the current study is the use of plant growth stimulating chromium-resistant bacteria in order to alleviate chromium toxicity and to expedite the growth of Zea mays plants with and without chromium stress by using pre-germination and post-germination inoculation treatments of Zea mays seeds.

\section{Experimental Procedures}

\section{Determining Chromium Resistance and Auxin Production of Bacterial Isolates}

To determine minimum inhibitory concentration and auxin production, six bacterial strains - Bacillus pumilus (ALa), Bacillus atrophaeus (BL2), Bacillus cereus (AR), Staphylococcus lentus (E3), T2aii, and W6ii - previously isolated and characterized by Fatima and Ahmed [11], Jamil [12], and Aslam [13] were selected for the current study. All bacterial isolates were grown to record minimum inhibitory concentration (MIC) of chromium using L-agar medium supplemented with chromium salt $\left(\mathrm{K}_{2} \mathrm{CrO}_{4}\right)$ in concentrations ranging $200-1200 \mu \mathrm{g} / \mathrm{ml}$. Bacteria were also checked for auxin production following Ahmed and Hasnain [14]. For auxin estimation, bacterial culture was centrifuged for $10 \mathrm{~min}$ at $10,000 \mathrm{rpm}$ and auxin production in the supernatant was estimated by using salkowski reagent. The optical density of the reaction mixture was taken at $535 \mathrm{~nm}$.

\section{Plant Inoculation Experiment}

On the basis of MIC of bacterial isolates, three concentrations of chromium were used for further experiment, i.e., 200,400 , and $600 \mu \mathrm{g} / \mathrm{ml}$. To examine the growth stimulatory effect of selected bacterial strains on the growth of corn plants grown under chromium stress, two different inoculating treatments were followed. One was pre-germination inoculation treatment in which corn seeds were treated with bacterial inoculum before germination, and the second was post-germination inoculation treatment in which corn seeds were treated with bacterial inoculum after germination.

Certified seeds of Zea mays var. DK-6103 were obtained from Punjab Seed Corporation, Lahore. For condition one, i.e., pre-germination inoculation treatment, seeds of Zea mays were surface-sterilized and treated with bacterial strains (Bacillus pumilus (ALa), Bacillus atrophaeus (BL2), Bacillus cereus (AR), Staphylococcus lentus (E3), T2aii, and W6ii) with and without chromium stress $(0,200,400$, and $600 \mu \mathrm{g} / \mathrm{ml})$ following Fatima and Ahmed [11]. For control treatment, seeds were treated with autoclaved distilled water for the same period of time. Seeds were also grown in the presence of chromium stress $(200,400$, and $600 \mu \mathrm{g} / \mathrm{ml})$ without bacterial treatment. Emergence of seeds for each treatment was observed regularly at the interval of eight hours. Post-germination inoculation treatment was also carried out. For post-germination inoculation treatment, seeds were inoculated with bacterial strains (Bacillus 
Table 1. Minimum inhibitory concentration (MIC) and auxin production of chromium-resistant bacterial isolates.

\begin{tabular}{|c|c|c|c|c|c|c|c|c|}
\hline \multirow{2}{*}{ Sr. \# } & \multirow{2}{*}{ Bacterial isolates } & \multicolumn{6}{|c|}{$\mathrm{K}_{2} \mathrm{CrO}_{4}(\mu \mathrm{g} / \mathrm{ml})$} & \multirow{2}{*}{ Auxin production } \\
\hline & & 200 & 400 & 600 & 800 & 1000 & 1200 & \\
\hline 1 & Bacillus pumilus (ALa) & +++ & +++ & +++ & +++ & +++ & - & +++ \\
\hline 2 & Bacillus atrophaeus (BL2) & +++ & +++ & +++ & +++ & ++ & - & +++ \\
\hline 3 & Bacillus cereus (AR) & +++ & +++ & +++ & +++ & ++ & - & +++ \\
\hline 4 & Staphylococcus lentus (E3) & +++ & +++ & +++ & ++ & +++ & - & +++ \\
\hline 5 & T2aii & +++ & +++ & +++ & + & - & - & +++ \\
\hline 6 & W6ii & +++ & +++ & +++ & + & - & - & +++ \\
\hline
\end{tabular}

Excellent growth (+++), Good growth (++), Slight growth (+), No growth (-)

pumilus (ALa), Bacillus atrophaeus (BL2), Bacillus cereus (AR), Staphylococcus lentus (E3), T2aii, and W6ii) after seed germination following Fatima and Ahmed [11]. Six seeds were sown per pot and four replicates were used for all treatments. After 28 days of seedling growth, corn seedlings were removed from their respective pots and various growth parameters of all treated plants were recorded, including shoot length, number of leaves, root length, and fresh weight. Biochemical parameters for all treatments were also studied, for which chlorophyll estimation and soluble protein content estimation was done by following Vaishnav et al. [15] and Lowry et al. [16], respectively.

\section{Statistical Analysis}

The data obtained were statistically analyzed using the software SPSS v.16 by applying Duncan's multiple range test.

\section{Results}

Determining Chromium Resistance and Auxin Production of Bacterial Isolates

Auxin production by all bacterial isolates (Bacillus pumilus (ALa), Bacillus atrophaeus (BL2), Bacillus cereus (AR), Staphylococcus lentus (E3), T2aii, and W6ii) was recorded (Table 1). Bacillus pumilus (ALa), Bacillus atrophaeus (BL2), Bacillus cereus (AR), and Staphylococcus lentus (E3) showed chromium resistance by exhibiting tremendous growth up to chromium stress of $1000 \mu \mathrm{g} / \mathrm{ml}$, while isolates T2aii and W6ii exhibited prominent growth up to $800 \mu \mathrm{g} / \mathrm{ml}$ (Table 1). All bacterial isolates were able to resist chromium ranging from $0-600 \mu \mathrm{g} / \mathrm{ml}$, so for further experiments, the concentration of chromium used was $600 \mu \mathrm{g} / \mathrm{ml}$.

\section{Plant Inoculation Experiment}

The symbiotic association between bacterial populations and plants exhibited significant growth of treated plants with improvement in various parameters. In the present work, several treatments of both bacterial inoculum (Bacillus pumilus (ALa), Bacillus atrophaeus (BL2), Bacillus cereus (AR), Staphylococcus lentus (E3), T2aii, and W6ii) and chromium stress 0, 200, 400, and $600 \mu \mathrm{g} / \mathrm{ml}$, were applied to Zea mays seeds either separately or in different combinations, i.e., control (without bacteria and chromium stress), with chromium stress only $(200,400$, and $600 \mu \mathrm{g} / \mathrm{ml})$, with bacteria only, with bacteria and chromium stress $(200,400$, and $600 \mu \mathrm{g} / \mathrm{ml})$ simultaneously in all combinations.

\section{Seed Emergence and Percentage Germination}

For pre-germination inoculation treatment, seed emergence of different treatments was recorded. No emergence was seen on day 1, while maximum emerged seeds were recorded on day 5. Data for days 3 (d3) and 5 (d5) showed that on d3, the maximum number of emerged seeds was 27 , as exhibited by T2aii treatment over non-inoculated control treatment, which showed 17 emerged seeds. However, under 200, 400, and $600 \mu \mathrm{g} / \mathrm{ml}$ of chromium stress, seeds inoculated with isolates T2aii, Bacillus cereus (AR), and T2aii have shown maximum emergence, i.e., up to 23,21 , and 20 seeds, respectively, over respective non-inoculated, chromium-stressed seeds showing 16, 15, and 14 emerged seeds, respectively. In pre-germination inoculation treatment, the percentage germination of inoculated seeds with different bacterial treatments has shown that without chromium stress, all the studied bacterial isolates (Bacillus pumilus (ALa), Bacillus atrophaeus (BL2), Bacillus cereus (AR), Staphylococcus lentus (E3), T2aii) exhibited $100 \%$ germination while control treatment exhibited $93.3 \%$ germination. Under chromium stress of $200 \mu \mathrm{g} / \mathrm{ml}$, bacterial treatments with Bacillus pumilus (ALa), Bacillus atrophaeus (BL2), and T2aii exhibited $96.6 \%$ germination over control treatment, which showed $90 \%$ germination. Under chromium stress of 400 and 600 $\mu \mathrm{g} / \mathrm{ml}$, bacterial inoculation with Bacillus pumilus (ALa) exhibited 100 and $96.6 \%$ germination respectively, when compared with respective control treatments, which have shown 90 and $86.6 \%$ germination under chromium 


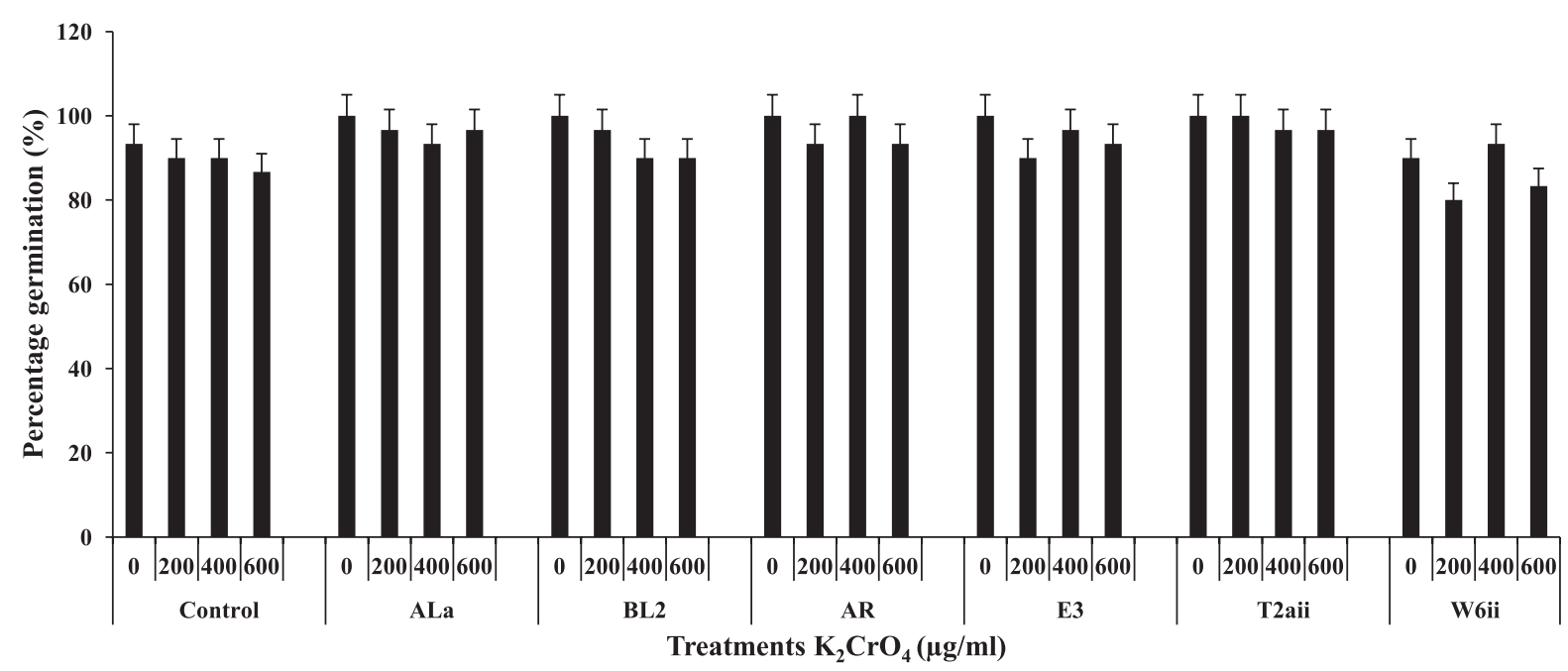

Fig. 1. Effect of bacterial inoculations with and without chromium stress $\left(\mathrm{K}_{2} \mathrm{CrO}_{4}(0,200,400\right.$, and $\left.600 \mu \mathrm{g} / \mathrm{ml})\right)$ on percentage germination of Zea mays in pre-germination inoculation treatment (C - Control; Bacillus pumilus (ALa), Bacillus atrophaeus (BL2), Bacillus cereus (AR), Staphylococcus lentus (E3), T2aii, and W6ii - Bacterial strains).

stress of 400 and $600 \mu \mathrm{g} / \mathrm{ml}$, in the case of the absence of bacterial treatments, respectively. As seeds were already germinated in post-germination inoculation treatment, so seed emergence and percentage germination of treated seeds were not observed (Fig. 1).

\section{Growth Parameters}

When plants were given bacterial treatments without chromium stress, pronounced increase in growth parameters were ascertained. In pre-germination inoculation treatment, Bacillus pumilus (ALa) showed maximum significant increase in shoot length of corn plants, i.e., 47.6\%, whereas isolates W6ii, T2aii, and Bacillus atrophaeus (BL2) have also shown significant increments in shoot length of treated plants $(44.3,14.4$, and $15 \%$, respectively) when compared with control plants, while in post-germination inoculation treatment, 63.9 and $52.2 \%$ increases were shown by Bacillus pumilus (ALa) and W6ii treatments, respectively, over control. Profound increment in root length was also recorded in pre-germination inoculation treatment when plants were treated with W6ii and Bacillus pumilus (ALa) showing 175 and 170.6\% enhancement in root length respectively when compared with control plants, whereas in post-germination treatment, Bacillus pumilus (ALa)- and W6ii-treated plants showed significant increment in root length, i.e., $182.7 \%$, over control (Fig. 2). Similarly, in pre-germination inoculation treatment, significant improvement in the number of leaves was shown by inoculated plants with bacterial treatments of Bacillus pumilus (ALa) and W6ii, i.e.,

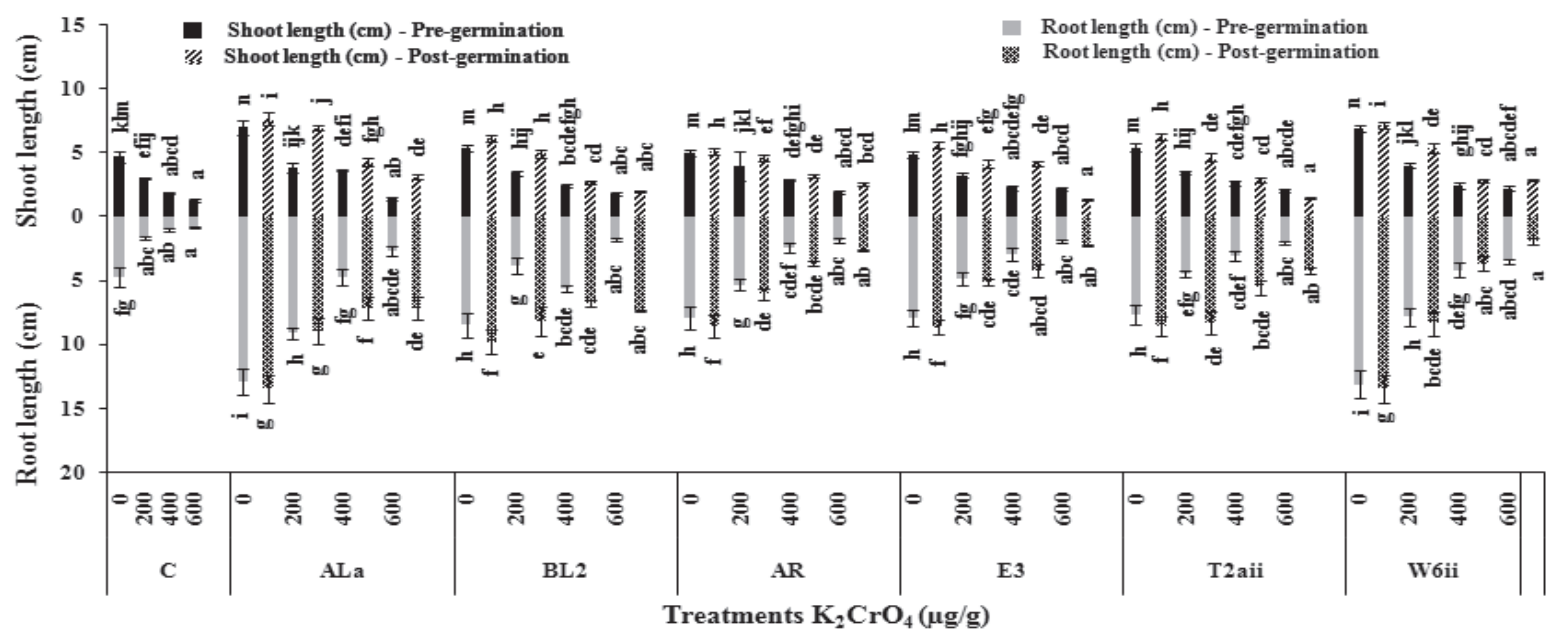

Fig. 2. Effect of bacterial inoculations with and without chromium stress $\left(\mathrm{K}_{2} \mathrm{CrO}_{4}(0,200,400\right.$, and $\left.600 \mu \mathrm{g} / \mathrm{g})\right)$ on shoot and root lengths of Zea mays in pre-germination and post-germination inoculation treatments; data represent mean of 24 replicates, with different letters indicating significant differences between treatments using Duncan's multiple range test $(\mathrm{P}=0.05)(\mathrm{C}$ - Control; Bacillus pumilus (ALa), Bacillus atrophaeus (BL2), Bacillus cereus (AR), Staphylococcus lentus (E3), T2aii, and W6ii - Bacterial strains). 


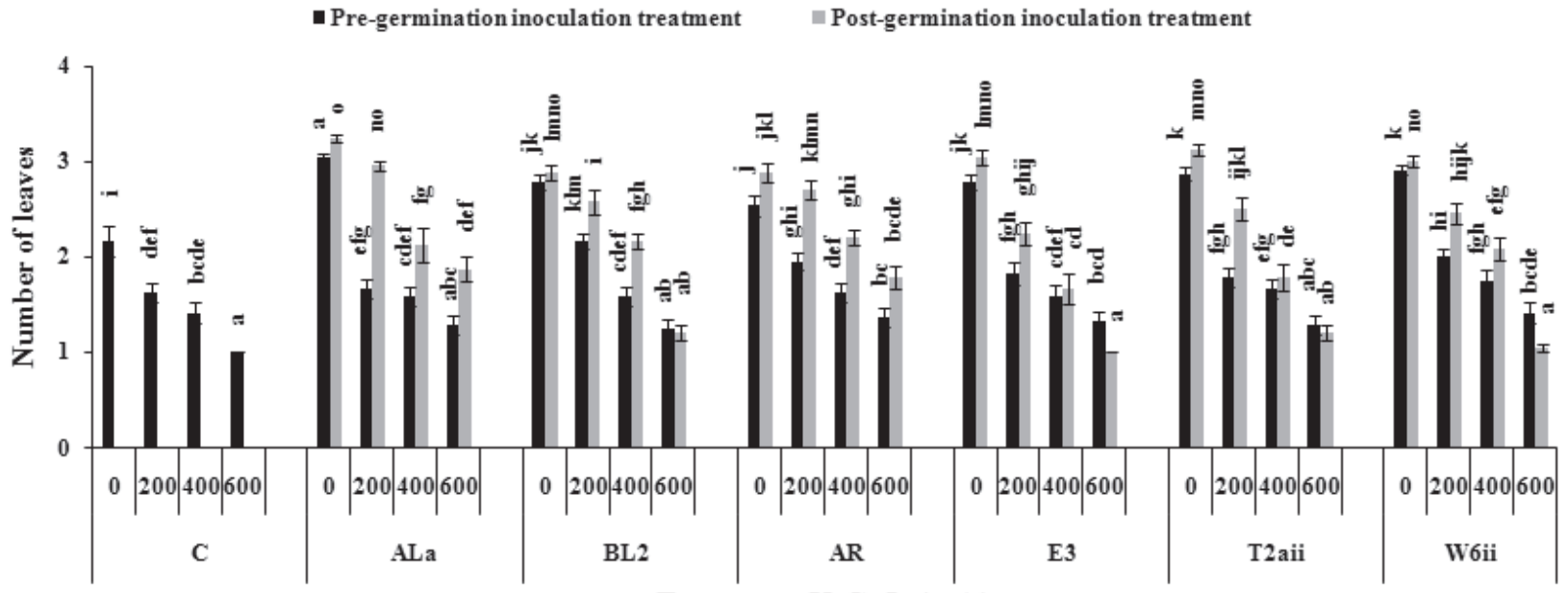

Treatments $\mathrm{K}_{2} \mathrm{CrO}_{4}(\mu \mathrm{g} / \mathrm{g})$

Fig. 3. Effect of bacterial inoculations with and without chromium stress $\left(\mathrm{K}_{2} \mathrm{CrO}_{4}(0,200,400\right.$, and $\left.600 \mu \mathrm{g} / \mathrm{g})\right)$ on number of leaves of Zea mays in pre-germination and post-germination inoculation treatments; data represent mean of 24 replicates, with different letters indicating significant differences between treatments using Duncan's multiple range test $(\mathrm{P}=0.05)(\mathrm{C}$ - Control; Bacillus pumilus (ALa), Bacillus atrophaeus (BL2), Bacillus cereus (AR), Staphylococcus lentus (E3), T2aii, and W6ii - Bacterial strains).

39.9 and $34.8 \%$, respectively, in comparison with control treatment, whereas Bacillus pumilus (ALa) and T2aii inoculations showed pronounced increases in the number of leaves in post-germination inoculation treatment, i.e., 48.6 and $45.1 \%$, respectively, as compared to control plants (Fig. 3). In addition, maximum significant improvement in fresh weight was recorded when maize plants were treated with Bacillus pumilus (ALa), exhibiting 98.9\% increment in pre-germination inoculation treatment. In the case of post-germination treatment, significant enhancement in fresh weight was recorded by W6ii- and Bacillus pumilus (ALa)- treated plants, i.e., 64.7 and $41.3 \%$ respectively, in comparison with control plants (Fig. 4). Plants grown in the absence of bacterial treatment under chromium stress of $200 \mu \mathrm{g} / \mathrm{g}$ exhibited 35.5, 25, 65.5, and 33.2\% decrease in shoot length, number of leaves, root length, and fresh weight, respectively, in contrast with non-inoculated and not stressed with chromium ions control treatments without stress. Similarly, plants exhibited significant decreases in shoot length, number of leaves, root length, and fresh weight, i.e., $60.1,34.6,78.5$, and $48.9 \%$, respectively, over control plants under chromium stress of $400 \mu \mathrm{g} / \mathrm{g}$ without bacterial treatment. In the presence of $600 \mu \mathrm{g} / \mathrm{g}$ chromium stress under non-inoculated conditions, 73.3, $82.6,73.2$, and $53.8 \%$ decreases were recorded in shoot length, root length, fresh weight, and number of leaves, respectively, over respective control plants without chromium stress and bacterial treatments (Figs 2, 3, and 4).

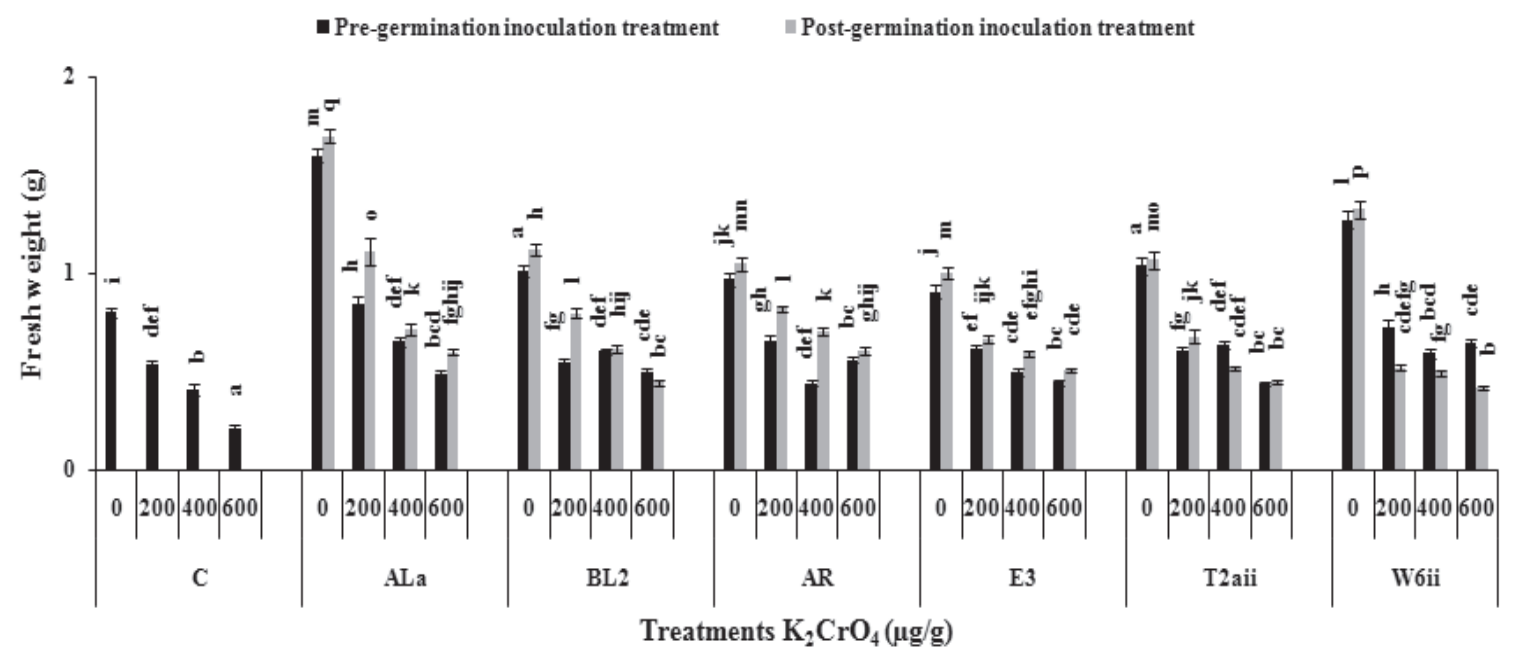

Fig. 4. Effect of bacterial inoculations with and without chromium stress $\left(\mathrm{K}_{2} \mathrm{CrO}_{4}(0,200,400\right.$, and $\left.600 \mu \mathrm{g} / \mathrm{g})\right)$ on fresh weight of Zea mays in pre-germination and post-germination inoculation treatments; data represent mean of 24 replicates, with different letters indicating significant differences between treatments using Duncan's multiple range test $(\mathrm{P}=0.05)(\mathrm{C}$ - Control; Bacillus pumilus (ALa), Bacillus atrophaeus (BL2), Bacillus cereus (AR), Staphylococcus lentus (E3), T2aii, and W6ii - Bacterial strains). 
When plants were inoculated with bacterial isolates under chromium stress, tremendous improvement in growth parameters was observed in comparison with respective control. Under $200 \mu \mathrm{g} / \mathrm{g}$ of chromium stress, inoculations with W6ii and Bacillus cereus (AR) significantly improved shoot length, i.e., 32.5 and 30.8\% respectively over control treatments in pre-germination treatment, while in post-germination inoculation treatment, profound results were obtained by Bacillus pumilus (ALa) and W6ii treatments exhibiting 129.3 and $77.9 \%$ enhancement respectively over non-inoculated control. Similarly, for pre-germination inoculation treatment, 456.2, 378.8, and 225.1\% increases were observed in root length with isolates Bacillus pumilus (ALa), W6ii, and Bacillus cereus (AR) respectively over control, whereas significant enhancement in root length was recorded by Bacillus pumilus (ALa), W6ii, and T2aii treatments (446.1, 409.3, and 406.2\%, respectively) in post-germination treatment in comparison with control plants (Fig. 2). In addition, after pre-germination inoculation, the number of leaves in treated plants were significantly improved with Bacillus atrophaeus (BL2) and W6ii treatment exhibiting 33.3 and 23\% increases, respectively, while in the case of post germination inoculation treatments, $82,66.6$, and $58.9 \%$ increments in the number of leaves was recorded in treated plants inoculated with Bacillus pumilus (ALa), Bacillus cereus (AR), and Bacillus atrophaeus (BL2) respectively, compared with respective control (Fig. 3). Also, Bacillus pumilus (ALa)- and W6ii-inoculated plants improved fresh weight by 57.6 and $34.8 \%$ in pre-germination inoculation treatment over control, whereas in postgermination inoculation treatment Bacillus pumilus (ALa), Bacillus cereus (AR), and Bacillus atrophaeus (BL2) treatments significantly improved fresh weight (106.8, 53.3, and $48.8 \%$ respectively) over non-inoculated control treatment (Fig. 4).
In pre-germination conditions, under $400 \mu \mathrm{g} / \mathrm{g}$ chromium stress, bacterial inoculations with Bacillus pumilus (ALa) and AR significantly improved shoot length (95.4 and 55.4\% respectively) compared to control, while in post-germination inoculation treatment, significant results were obtained by Bacillus pumilus (ALa) and Staphylococcus lentus (E3) treatments exhibiting 129.4 and $124.5 \%$ enhancement in shoot length respectively when compared with control plants. Significant increases in root length were recorded in Bacillus atrophaeus (BL2), Bacillus pumilus (ALa), and W6ii treatments in pre-germination inoculation treatment (446.5, 365.7, and 306.5\% respectively), whereas in post-germination treatment plants inoculated with Bacillus pumilus (ALa) and Bacillus atrophaeus (BL2) have shown 596.3 and 567.6\% enhancement in root length respectively, in contrast with non-inoculated control plants under respective chromium stress (Fig. 2). In pre-germination treatment, a significant increase in the number of leaves was recorded for W6ii- and T2aii-inoculated plants, i.e., 23.5 and 17.6\% respectively, whereas in post-germination inoculation treatment the leaves have been shown to be more numerous (55.8 and 52.9\% increase) per plant when plants were treated with Bacillus cereus (AR) and Bacillus atrophaeus (BL2) respectively, in comparison with control treatments (Fig. 3). Prominent increases in fresh weight were ascertained in pre-germination treatment, when corn seedlings were treated with Bacillus pumilus (ALa) and T2aii showing 59.6 and $54.2 \%$ increase respectively when compared with control, while increment in fresh weight were recorded as a result of treatments with Bacillus pumilus (ALa) and Bacillus cereus (AR) in post-germination treatment, i.e., 75.3 and $71.9 \%$ respectively, in contrast with control (Fig. 4).

Under chromium stress of $600 \mu \mathrm{g} / \mathrm{g}$ in pregermination inoculation treatment, plants inoculated

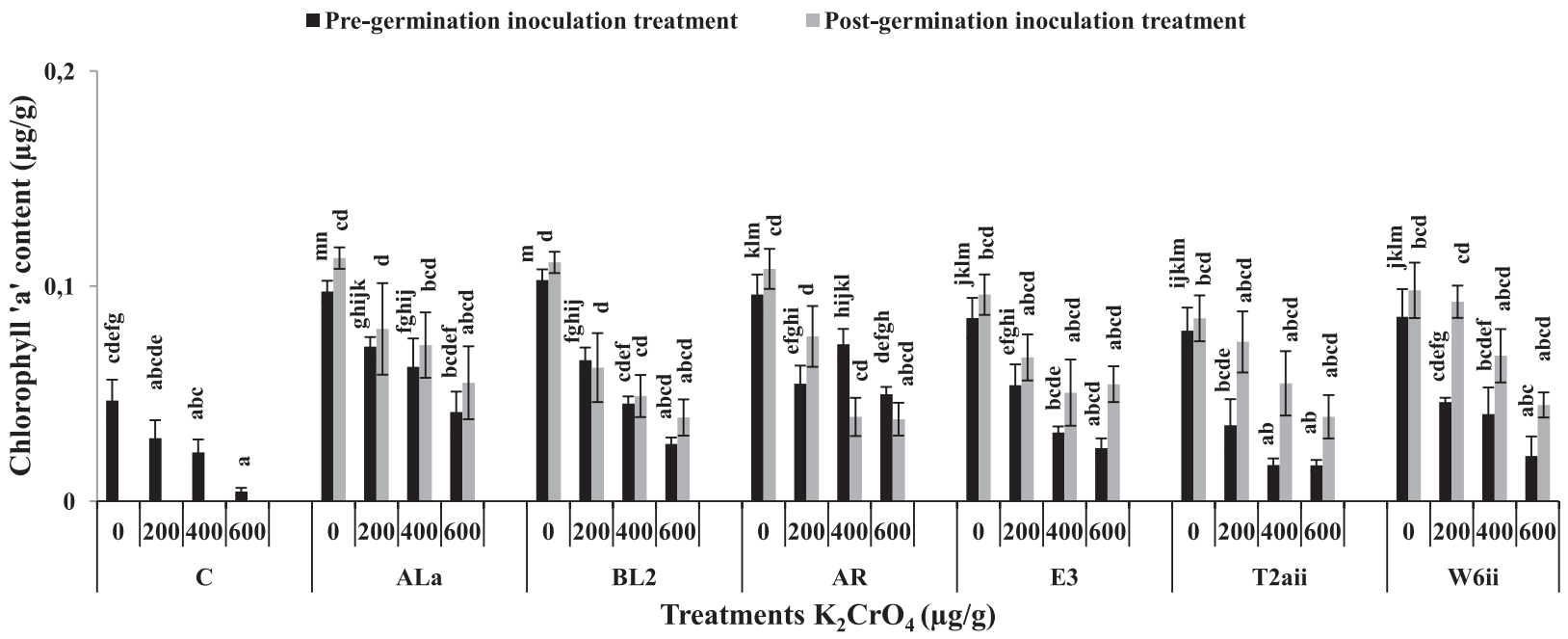

Fig. 5. Effect of bacterial inoculations with and without chromium stress $\left(\mathrm{K}_{2} \mathrm{CrO}_{4}(0,200,400\right.$, and $\left.600 \mu \mathrm{g} / \mathrm{g})\right)$ on chlorophyll 'a' content of Zea mays in pre-germination and post-germination inoculation treatments; data represent mean of 24 replicates, with different letters indicating significant differences between treatments using Duncan's multiple range test $(\mathrm{P}=0.05)(\mathrm{C}$ - Control; Bacillus pumilus $(\mathrm{ALa})$, Bacillus atrophaeus (BL2), Bacillus cereus (AR), Staphylococcus lentus (E3), T2aii, and W6ii - Bacterial strains). 


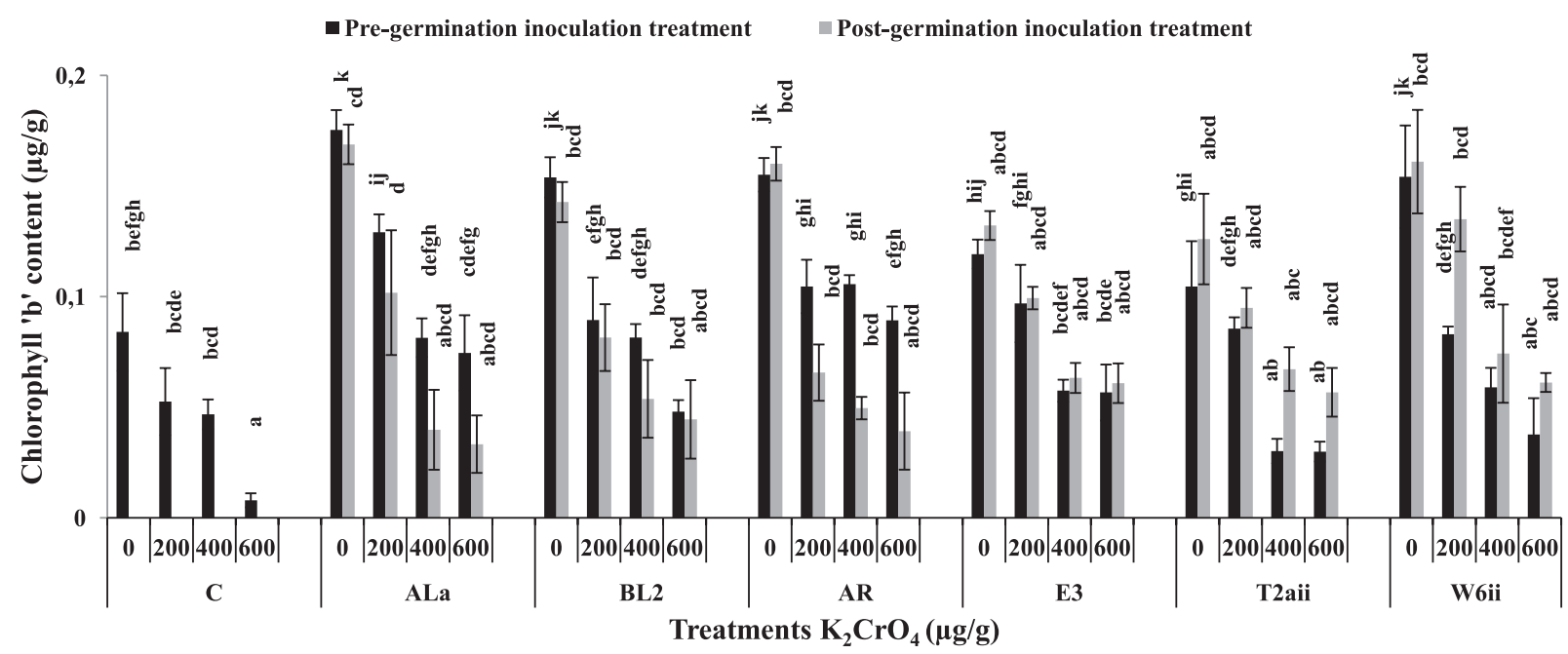

Fig. 6. Effect of bacterial inoculations with and without chromium stress $\left(\mathrm{K}_{2} \mathrm{CrO}_{4}(0,200,400\right.$, and $\left.600 \mu \mathrm{g} / \mathrm{g})\right)$ on chlorophyll 'b' content of Zea mays in pre-germination and post-germination inoculation treatments; data represent mean of 24 replicates, with different letters indicating significant differences between treatments using Duncan's multiple range test $(\mathrm{P}=0.05)(\mathrm{C}$ - Control; Bacillus pumilus (ALa), Bacillus atrophaeus (BL2), Bacillus cereus (AR), Staphylococcus lentus (E3), T2aii, and W6ii - Bacterial strains).

with W6ii and Staphylococcus lentus (E3) have shown 79 and $72.5 \%$ increases in shoot length respectively, while in the case of post-germination treatment, tremendous increase was recorded in shoot length in Bacillus pumilus (ALa)-, W6ii-, and Bacillus cereus (AR)- inoculated plants $(149.6,133$, and $105.3 \%$ respectively) over control treatment. Root length of treated plants was observed in pre-germination inoculation treatment in which plants inoculated with W6ii and Bacillus pumilus (ALa) exhibited 321.3 and $224.2 \%$ increases, respectively, while in the case of post-germination treatment, significant enhancement in root length was observed in corn seedlings treated with Bacillus atrophaeus (BL2) and Bacillus pumilus (ALa) - up to 783.5 and $768.1 \%$ respectively, in contrast with control treatment (Fig. 2). Similarly, in pre-germination treatment, W6ii, Bacillus cereus (AR), and Staphylococcus lentus (E3) treatments exhibited 41.6, 38.1, and 32.9\% enhancement in number of leaves of treated plants respectively as regards control plants. On the other hand, inoculated plants of postgermination treatment treated with Bacillus pumilus (ALa) and Bacillus cereus (AR) significantly enhanced the number of leaves, i.e., 87.5 and $41.4 \%$ respectively, over control treatment (Fig. 3). Similarly, plants treated with isolates W6ii, Bacillus cereus (AR), and Bacillus atrophaeus (BL2) exhibited significantly improved fresh weight (up to $200.9,161$, and $133.3 \%$ respectively) while in the case of post-germination inoculation treatment, 181.5 and $179.4 \%$ increases were recorded in fresh weight when plants were treated with Bacillus cereus (AR) and Bacillus pumilus (ALa) over control plants (Fig. 4).

\section{Biochemical Parameters}

When plants were grown without chromium stress, bacterial treatments with Bacillus pumilus (ALa), Bacillus atrophaeus (BL2), Bacillus cereus (AR), Staphylococcus lentus (E3), T2aii, and W6ii exhibited significant enhancement in chlorophyll content of inoculated plants given pre-germination inoculation treatment. Pronounced enhancement in chlorophyll 'a,' 'b,' and total chlorophyll content was recorded by Bacillus pumilus (ALa) treatment (108.6\%), while bacterial treatment with Bacillus cereus (AR) also significantly improved chlorophyll 'a,' 'b,' and total chlorophyll content (105.3, 84.6 and 92.1\% respectively) as compared to respective control treatment. Significant enhancement in chlorophyll 'a,' 'b,' and total chlorophyll content was exhibited by Bacillus pumilus (ALa) treatment (142.4, 134.6, and 138.2\% respectively) in post-germination treatment, while Bacillus atrophaeus (BL2)-inoculated plants exhibited 137.7, 110.1, and 90.5\% enhancement in chlorophyll 'a,' 'b,' and total chlorophyll content respectively over respective control plants. Similarly, the effect of bacterial inoculations on soluble protein content of treated and non-treated plants was also observed (Figs. 5-7). In pre-germination treatment, elevated concentration of protein content was noted in Bacillus pumilus (ALa)-treated plants (105.9\%), while 90.3\% enhancement was recorded in bacterial treatments with Bacillus atrophaeus (BL2) and Staphylococcus lentus (E3) when compared with control plants. On the other hand, in post-germination treatment, maximum pronounced enhancement in soluble protein content was observed in Bacillus pumilus (ALa)-treated plants (79.4\%) over control. Similarly, treatments with Staphylococcus lentus (E3), Bacillus atrophaeus (BL2), and Bacillus cereus (AR) exhibited significant enhancement in protein content $(65.3,44.9$, and $45 \%$ respectively) over control plants (Fig. 8).

Plants grown under chromium stress without bacterial treatments were analyzed and their chlorophyll 'a,' 'b,' and total chlorophyll contents were contrasted with control treatments without chromium stress. Under $200 \mu \mathrm{g} / \mathrm{g}$ of chromium stress, $37.5 \%$ reduction 


\section{- Pre-germination inoculation treatment $\quad$ Post-germination inoculation treatment}

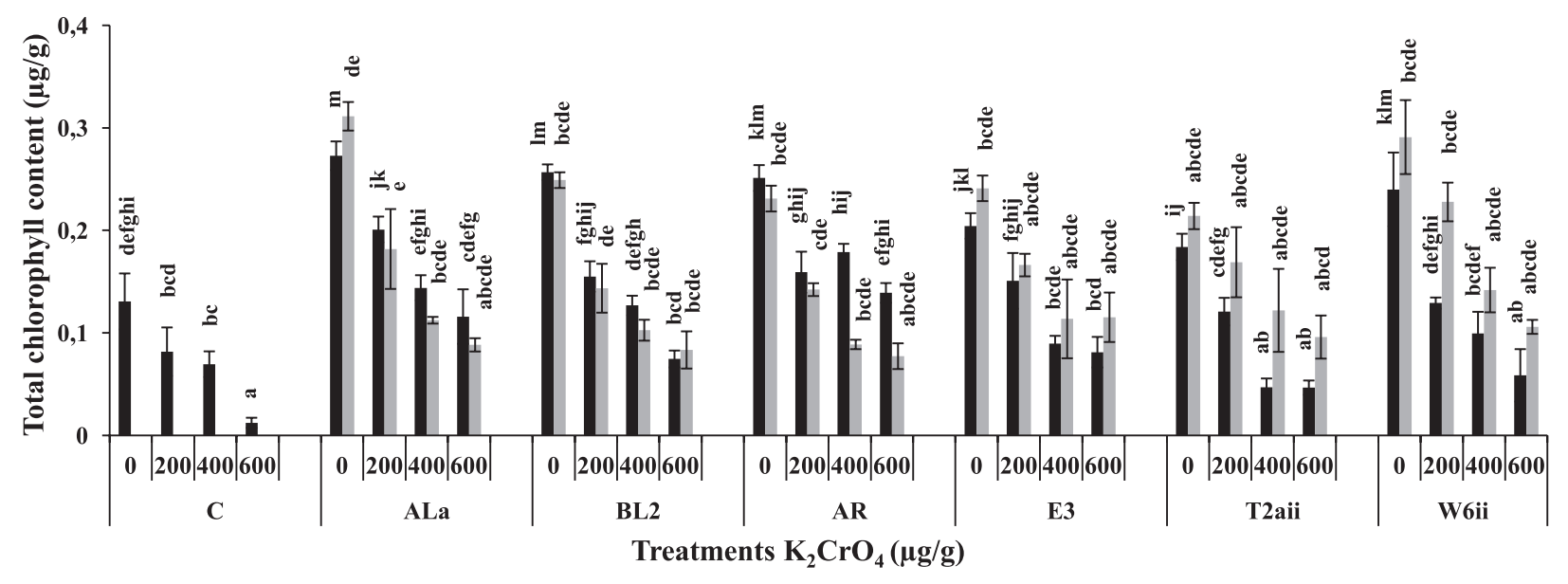

Fig. 7. Effect of bacterial inoculations with and without chromium stress $\left(\mathrm{K}_{2} \mathrm{CrO}_{4}(0,200,400\right.$, and $\left.600 \mu \mathrm{g} / \mathrm{g})\right)$ on total chlorophyll content of Zea mays in pre-germination and post-germination inoculation treatments; data represent mean of 24 replicates, with different letters indicating significant differences between treatments using Duncan's multiple range test $(\mathrm{P}=0.05)(\mathrm{C}$ - Control; Bacillus pumilus (ALa), Bacillus atrophaeus (BL2), Bacillus cereus (AR), Staphylococcus lentus (E3), T2aii, and W6ii - Bacterial strains).

in chlorophyll 'a,' 'b,' and total chlorophyll content was observed over control plants. Under $400 \mu \mathrm{g} / \mathrm{g}$ chromium stress, chlorophyll 'a,' 'b,' and total chlorophyll significantly decreased (51.6, 44.3, and 46.3\% respectively) over respective control plants. Similarly, plants grown under $600 \mu \mathrm{g} / \mathrm{g}$ chromium stress have also shown significant decreases in chlorophyll 'a,' 'b,' and total chlorophyll (90.6\%) over control treatment (Figs. 5-7). Total soluble protein content was compared with respective non-inoculated control plants, and 8.1, 35.4, and $51.4 \%$ decreases in protein content were observed by the plants grown under 200, 400, and $600 \mu \mathrm{g} / \mathrm{g}$ of chromium stress, respectively, over control (Fig. 8).
When plants were grown with bacterial treatments under chromium stress, pronounced increases in biochemical parameters were observed when compared with their respective control treatments. In pregermination inoculation treatment, under $200 \mu \mathrm{g} / \mathrm{g}$ of chromium stress Bacillus pumilus (ALa)-treated plants exhibited significant enhancement, i.e., 145.8, 145.7, and $145.8 \%$ in chlorophyll 'a,' 'b,' and total chlorophyll respectively over control plants. Similarly, plants treated with Bacillus cereus (AR) exhibited 86.9, 99.1, and 94.8\% enhancement in chlorophyll 'a,' 'b,' and total chlorophyll over control, whereas in post-germination inoculation treatment treated plants with isolate W6ii showed significant enhancement in chlorophyll 'a,' 'b,' and total

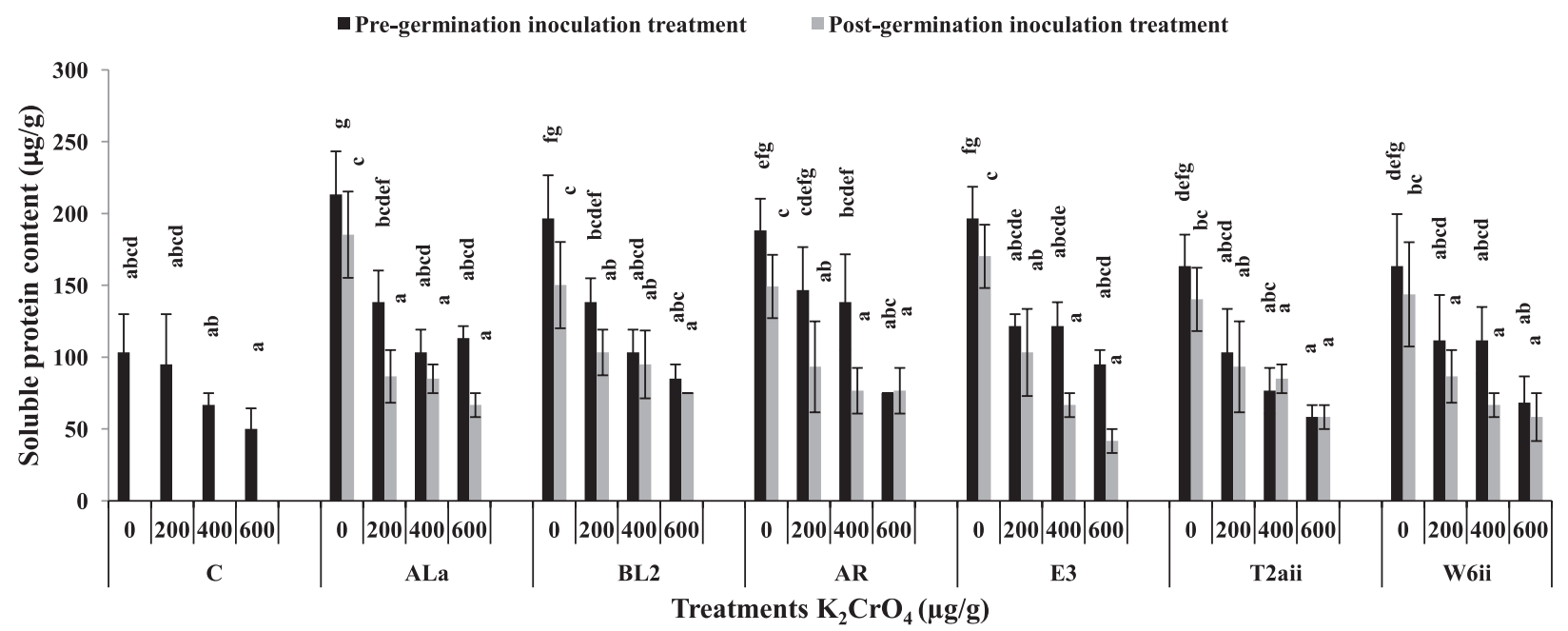

Fig. 8. Effect of bacterial inoculations with and without chromium stress $\left(\mathrm{K}_{2} \mathrm{CrO}_{4}(0,200,400\right.$, and $\left.600 \mu \mathrm{g} / \mathrm{g})\right)$ on soluble protein content of Zea mays in pre-germination and post-germination inoculation treatments; data represent mean of 24 replicates, with different letters indicating significant differences between treatments using Duncan's multiple range test $(\mathrm{P}=0.05)(\mathrm{C}$ - Control; Bacillus pumilus (ALa), Bacillus atrophaeus (BL2), Bacillus cereus (AR), Staphylococcus lentus (E3), T2aii, and W6ii - Bacterial strains). 
chlorophyll ( $216.8,158$, and $179.7 \%$ respectively), while $174.3,93.8$, and $122.6 \%$ increase in chlorophyll 'a,' 'b,' and total chlorophyll was recorded when plants were treated with Bacillus pumilus (ALa) respectively over control plants (Fig. 5-7). Total soluble protein content of pre-germination treatment was analyzed and a $54.4 \%$ increase was recorded in Bacillus cereus (AR)-inoculated plants while a $45.6 \%$ increase was observed in both Bacillus pumilus (ALa) and Bacillus atrophaeus (BL2) treatments over control. In the case of post-germination inoculation treatment, Bacillus atrophaeus (BL2) and Staphylococcus lentus (E3) treatments showed 8.5 and $8.9 \%$ enhancement in soluble protein content respectively over respective control (Fig. 8).

Under chromium stress of $400 \mu \mathrm{g} / \mathrm{g}$, Bacillus cereus (AR) showed maximum enhancement in chlorophyll 'a,' 'b,' and total chlorophyll (221.3, 126, and 156.8\% respectively) in pre-germination inoculation treatment when compared with control. Similarly, plants treated with Bacillus pumilus (ALa) have also significantly improved chlorophyll 'a,' 'b,' and total chlorophyll (176.7, 74.1, and $107.5 \%$ respectively) when compared to control plants. In post-germination inoculation treatment, inoculation with Bacillus pumilus (ALa) exhibited 199.3, 58.7, and $104.5 \%$ increments in chlorophyll 'a,' 'b', and total chlorophyll over control. Similarly, T2aii-treated plants caused significant improvement in chlorophyll 'a,' 'b,' and total chlorophyll (142.5, 43.7, and 75.9\% respectively) when compared with control plants (Figs. 5-7). Soluble protein content in pre-germination inoculation treatment was observed, and 107.5, 82.5, and $67.5 \%$ increases were recorded for Bacillus cereus (AR), Staphylococcus lentus (E3), and W6ii treatments, respectively, over control while in post-germination inoculation treatment a $42.5 \%$ increase was observed in Bacillus atrophaeus (BL2)-treated plants and a 27.5\% increase was recorded for both Bacillus pumilus (ALa) and T2aii treatments over respective control plants (Fig. 8).

Under chromium stress of $600 \mu \mathrm{g} / \mathrm{g}$, chlorophyll content of treated plants in pre-germination inoculation treatment significantly enhanced chlorophyll 'a,' 'b,' and total chlorophyll in Bacillus cereus (AR)-treated plants (1036.2\%) over respective control treatment. Similarly, an $847.7 \%$ increase was recorded for Bacillus pumilus (ALa)-treated plants, in chlorophyll 'a,' 'b,' and total chlorophyll when compared with control plants, whereas in post-germination treatment, pronounced enhancement in chlorophyll 'a,' 'b,' and total chlorophyll was seen in the case of W6ii-inoculated plants exhibiting 922.8, 160.2 , and $765.6 \%$ increase respectively, over control. Similarly, T2aii-treated plants have shown 796.7, 146.8, and $684.2 \%$ increase in chlorophyll 'a,' 'b,' and total chlorophyll, in contrast with control plants (Figs 5-7). In pre-germination treatment, corn seedlings inoculated with Bacillus pumilus (ALa), Staphylococcus lentus (E3) and Bacillus atrophaeus (BL2) exhibited 126.6, 90, and $70 \%$ enhancement in protein content, respectively, over control while in post-germination inoculation treatment, significant improvement in soluble protein content was noted for treatments with AR and Bacillus atrophaeus (BL2) (53.3 and 50\% respectively) over respective control treatment (Fig. 8).

\section{Discussion}

Heavy metal pollution in our environment is a serious issue that must be solved for the survival of the ecological community and for public safety. Due to industrialization, large amounts of heavy metals are released in the environment by various anthropogenic activities, which became an exigent matter over the past few decades [17]. In the present study, six efficient auxin-producing, chromium-resistant bacterial strains were used for the plant inoculation experiment (Table 1). Data recorded for percentage germination of inoculated and non-inoculated seeds demonstrated that chromium toxicity caused a reduction in sugar formation, which is crucial for the development of seed embryo, leading to the immobility of $\alpha$ - and $\beta$-amylase and thus inhibited seed germination [18]. Inoculation treatments of all the bacterial isolates used (Bacillus pumilus (ALa), Bacillus atrophaeus (BL2), Bacillus cereus (AR), Staphylococcus lentus (E3), W6ii, and T2aii) caused improvement in germination of corn seeds, which may be due to the production of gibberellins, which help enhance activity of $\alpha$ - and $\beta$-amylase, nuclease, and protease, which are involved in efficient germination of seeds and in the hydrolysis and assimilation of the starch [19]. It might be possible that growth-stimulating bacteria used for the inoculation experiment are involved in efficient production of IAA (indole-3-acidic acid), which ultimately helped in significant improvement in growth parameters, i.e., shoot length, fresh weight, number of leaves, and root length. Cell walls of treated plants became loose, assisted by IAA and bacteria, and absorb different substances released from plant roots. An amino acid, tryptophan, is responsible for the regulation of IAA production [20]. It has been reported that ethylene facilitated several physiological changes in plants, but the surplus production of ethylene caused retardation in growth of treated plants. Plant growth-promoting bacteria produce ACC-deaminase enzymes that regulate hydrolysis of ACC by converting it into $\alpha$-ketobutyrate and ammonia. Roots of treated plants have shown growth enhancement as they get sufficient nutrients, including nitrogen, which has been produced from this ammonia [21]. Our results have shown that toxicity of chromium decreased the number of plant leaves and also caused their chlorosis and reduction in size and leaf area.

Chromium-resistant bacteria with growth stimulation potential are able to convert Cr (VI) to $\mathrm{Cr}$ (III), which is immobile and insoluble in water so it is less toxic for plants [22]. Dhal and coworkers (2013) proposed that the capability of hexavalent chromium to reduce from a more toxic to a less toxic state varies from bacterial species to species depending upon their nutrient absorbance 
ability and other activities that directly influence plant tolerance against $\mathrm{Cr}$ (VI) [23]. Similar findings of growth potential of PGPB under stressed conditions have also been reported by other researchers [11, 17, 24, 25]. Bacterial treatments significantly improved chlorophyll and soluble protein content in Zea mays plants grown under chromium stress. In 2016, Premachandra et al. reported that significant enhancement in chlorophyll content of treated plants is due to the process of nitrogen fixation (BNF). As nitrogen is a vital constituent in the chlorophyll molecule, growth-stimulating bacteria use free nitrogen from the atmosphere and then convert it into ammonia and some other useful forms. The mechanism of biological nitrogen fixation is facilitated by nitrogenase. Various studies have revealed that bacteria associated with treated plants during inoculation treatments produce siderophores efficiently, which stimulate growth of the plants and enhance the nutritional value of treated plants, leading to the high chlorophyll and soluble protein content that helps in photosynthesis [8]. Similarly, Vafadar and other workers [26] also reported significant increases in chlorophyll 'a,' 'b,' and total chlorophyll in treated plants of Stevia rebaudiana by treating with growth-stimulating bacterial isolates such as Azotobactor chroococcum, Bacillus polymixa, and Pseudomonas putida.

In the current study, the most significant improvements in plant growth parameters and chlorophyll ' $a$ ' and ' $b$ ' content were recorded in postgermination treatment, while protein content was enhanced in those plants that were given pre-germination inoculation treatment. The possible explanation might be that in post-germination inoculation treatment, bacteria had a better chance of sticking on seeds along with radicle since the exposed surface area of seeds was increased after germination with radicle and plumule. Similarly, in post-germination inoculation treatments, seeds were transferred into their respective labelled pots immediately after inoculations, thus all the bacteria were viable and fully efficient. As a result, as soon as they get soil medium, they started their activity and got involved in several other mechanisms to enhance plant growth (i.e., bacteria "need not wait" for seed germination to express their activity as in pre-germination inoculation treatment). Another reason may be that the growth phase of bacteria played a key role in determining their plant growth promotional potential, so in the case of post-germination inoculation treatment the maximum percentage of bacteria are in their logarithmic or stationary phase, thus exhibiting maximum growth promotional activity. On the other hand, in pre-germination inoculation treatment, during germination of seeds some of the bacteria may die and some may have slowed down their activity due to some environmental changes, hence after getting transferred into the soil medium the availability of bacteria with maximum growth-promoting activity was less. Thus, growth parameters of plants were less improved over control as compared to post-germination inoculation treatment. And thus the present study clearly showed that the time of bacterial treatment of seeds during plant-microbe interaction studies for plant growth improvement is very important and should be considered a primary factor affecting plant growth under chromium stress. Further research is proposed to better know the detailed mechanisms of pre-germination and postgermination inoculation treatments to ameliorate the growth potential of plants under metal stressed conditions.

\section{Conclusions}

Our current study provides new insight toward the green approach in the reduction of chromium toxicity in plants grown in chromium-contaminated areas by applying chromium-resistant PGPB. Bacterial isolates used for the present study have shown growth promotional potential. The study clearly indicated that post-germination inoculation treatment helped reduce chromium toxicity and improve plant growth in terms of growth parameters with the exception of protein content. Thus, seeds should be given post-germination inoculation treatments for bacterial bioremediation to get maximum growth improvement in plants in chromiumcontaminated areas. In the present work, the bacterial strain Bacillus pumilus (ALa) has proven most efficient in all studied parameters out of the six isolates used. Hence, the present investigation supports the idea that bioremediation can be used as the best alternative in place of synthetic and chemical approaches to mitigate metal toxicity and to promote plant growth.

\section{Acknowledgements}

This work was supported by a financial grant from the University of the Punjab, Lahore, Pakistan.

\section{Conflict of Interest}

The authors declare no conflict of interest.

\section{References}

1. TCHOUNWOU P.B., YEDJOU C.G., PATLOLLA A.K. AND SUTTON D.J. Heavy metal toxicity and the environment. In: Molecular, Clinical and Environmental Toxicology, Springer Basel, 101, 133, 2012.

2. SAYEL H., JOUTEY N.T., BAHAFID W., EL GHACHTOULI N. Chromium resistant bacteria: impact on plant growth in soil microcosm. Arch. Environ. Prot. 40 (2), 81, 2014.

3. SARWAR N., IMRAN M., SHAHEEN M.R., ISHAQ W., KAMRAN A., MATLOOB A., REHIM A., HUSSAIN S. Phytoremediation strategies for soils contaminated with heavy metals: modifications and future perspectives. Chemosphere. 171, 710, 2016. 
4. KAMRAN M.A., BIBI S., XU R.K., HUSSAIN S., MEHMOOD K., CHAUDHARY H. Phyto-extraction of chromium and influence of plant growth promoting bacteria to enhance plant growth. J. Geochem. Explor. doi: org/10.1016/j.gexplo.2016.09.005, 2016.

5. ISLAM F., YASMEEN T., ARIF M.S., RIAZ M., SHAHZAD S.M., IMRAN Q. ALI I. Combined ability of chromium $(\mathrm{Cr})$ tolerant plant growth promoting bacteria (PGPB) and salicylic acid (SA) in attenuation of chromium stress in maize plants. Plant Physiol. Biochem.108, 456, 2016.

6. AHEMAD M. Enhancing phytoremediation of chromiumstressed soils through plant-growth-promoting bacteria. Genet. Eng. Biotechnol. J. 13 (1), 51, 2015.

7. SANTOYO G., MORENO-HAGELSIEB G., DEL CARMEN OROZCO-MOSQUEDA M., GLICK B.R. Plant growth-promoting bacterial endophytes. Microbiol. Res. 183, 92, 2016.

8. DE-SOUZA R. AMBROSINI A. PASSAGLIA L.M. Plant growth-promoting bacteria as inoculants in agricultural soils. Genet. Mol. Biol. 38 (4), 401, 2015.

9. BENSIDHOUM L.. NABTI E.. TABLI N.. KUPFERSCHMIED P.. WEISS A.. ROTHBALLER M.. SCHMID M.. KEEL C.. HARTMANN A. Heavy metal tolerant Pseudomonas protegensisolates from agricultural well water in northeastern Algeria with plant growth promoting, insecticidal and antifungal activities. Eur. J. Soil Biol.75, 38, 2016.

10. MEHNAZ S., KOWALIK T., REYNOLDS B., LAZAROVITS G. Growth promoting effects of corn (Zea mays) bacterial isolates under greenhouse and field conditions. Soil. Biol. Biochem.42 (10), 1848, 2010.

11. FATIMA H., AHMED A. How chromium-resistant bacteria can improve corn growth in chromium-contaminated growing medium. Pol. J. Environ. Stud. 25 (6), 2357, 2016.

12. JAMIL N. Enzymatic and biochemical analysis of inoculated and non-inoculated plants grown under chromium stress using Triticum aestivum., University of the Punjab, Lahore; 58, Pakistan, 2016.

13. ASLAM T. Screening for lead-resistant PGPR and their application to reduce lead toxicity in plants., University of the Punjab, Lahore; 39, Pakistan, 2015.

14. AHMED A., HASNAIN S. Auxin producing Bacillus sp.: Auxin quantification and effect on the growth Solanum tuberosum. Pure Appl. Chem. 82 (1), 313, 2010.

15. VAISHNAV A., KUMARI S., JAIN S., VARMA, CHOUDARY D.K. Putative bacterial volatile-mediated growth in soyabean (Glycine max L Merrill) and expression of induced proteins under salt stress. J. Appl. Microbiol. 199, 539, 2015.
16. LOWRY O.H., RESEBROUGH N.J., FARR A.L. Protein measurement with the folin-phenol reagent. J. Biol. Chem. 193, 265, 1951.

17. SOBARIU D.L., FERTU D.I.T., DIACONU M., PAVEL L.V., HLIHOR R.M., DRAGOI E.N., CURTEANU S., LENZ M., CORVINI P.F.X., GAVRILESCU M. Rhizobacteria and plant symbiosis in heavy metal uptake and its implications for soil bioremediation. N. Biotechnol. doi: org/10.1016/j.nbt.2016.09.002, 2016.

18. SHAHID M., SHAMSHAD S., RAFIQ M., KHALID S., BIBI I., NIAZI N.K., DUMAT, C.RASHID M.I. Chromium speciation, bioavailability, uptake, toxicity and detoxification in soil-plant system: A review. Chemosphere. 178, 513, 2017.

19. NOUMAVO P.A., AGBODJATO N.A., BABA-MOUSSA F., ADJANOHOUN, A., BABA-MOUSSA L. Plant growth promoting rhizobacteria: Beneficial effects for healthy and sustainable agriculture. Afr. J. Biotechnol. 15 (27), 1452, 2016.

20. PREMACHANDRA D., HUDEK L., BRAU L. Bacterial modes of action for enhancing of plant growth. J. Biotechnol. Biomater.6 (3), 1, 2016.

21. ULLAH A., HENG S., MUNIS M.F.H., FAHAD S., YANG X. Phytoremediation of heavy metals assisted by plant growth promoting (PGP) bacteria: a review. Environ. Exper. Bot.117, 28, 2015.

22. MAQBOOL Z., ASGHAR H.N., SHAHZAD T., HUSSAIN S., RIAZ M., ALI S., ARIF M.S., MAQSOOD M. Isolating, screening and applying chromium reducing bacteria to promote growth and yield of okra (Hibiscus esculentus L.) in chromium contaminated soils. Ecotoxicol. Environ. Saf.114, 343, 2015.

23. DHAL B. THATOI H.N., DAS N.N., PANDEY B.D. Chemical and microbial remediation of hexavalent chromium from contaminated soil and mining/ metallurgical solid waste: a review. J. Hazard. Mater.250, $272,2013$.

24. VIRUEL E., ERAZZÚ L.E., MARTÍNEZCALSINA, L., FERRERO M.A., LUCCA M.E., SIÑERIZ F. Inoculation of maize with phosphate solubilizing bacteria: effect on plant growth and yield. J. Soil Sci. Plant. Nutr. 14 (4), 819, 2014.

25. HUANG Y., FENG H., LU H., ZENG Y. A thorough survey for Cr-resistant and/or-reducing bacteria identified comprehensive and pivotal taxa. Int. Biodeterior. Biodegradation. 117, 22, 2017.

26. VAFADAR F., AMOOAGHAIE R., OTROSHY M. Effects of plant-growth-promoting rhizobacteria and arbuscular mycorrhizal fungus on plant growth, stevioside, NPK, and chlorophyll content of Stevia rebaudiana. J. Plant Interact. 9 (1), 128, 2014. 
